\title{
EL APRENDIZAJE POR DESCUBRIMIENTO. UN CAMBIO METODOLÓGICO PARA APRENDER DIDÁCTICA DE LA HISTORIA ${ }^{1}$
}

\section{DISCOVERY LEARNING. A METHODOLOGICAL CHANGE TO LEARN DIDACTICS OF HISTORY}

\author{
https://doi.org/10.15304/ie.30.6861
}

\author{
María Pilar Molina Torres \\ Universidad de Córdoba \\ pilar.molina@uco.es
}

\section{RESUMEN}

El presente trabajo muestra una experiencia didáctica cuyo principal objetivo se ha centrado en incorporar dinámicas de trabajo en equipo con el alumnado de cuarto curso de Educación Primaria y en adquirir las competencias educativas para aproximarse al origen y evolución del ser humano, a las formas de vida prehistóricas, y a las manifestaciones culturales y artísticas creadas por aquellos primeros humanos, en el período más largo de la Humanidad. Mediante la implementación de una metodología investigativa se analizaron los acontecimientos históricos relacionados con el periodo que abarca desde el Paleolítico hasta la Edad de los Metales y se buscó fortalecer las capacidades históricas de los/as estudiantes con la realización de ejercicios que trabajaron el tiempo histórico y el espacio cronológico donde se desarrollaron los hechos más relevantes de la Prehistoria. Los resultados de su puesta en práctica mostraron a un alumnado capaz de analizar e interpretar acontecimientos con coherencia y perspectiva históricas. Las conclusiones que arroja la propuesta destacan por el trabajo cooperativo como instrumento para garantizar el aprendizaje de indagación y el conocimiento del patrimonio prehistórico.

Palabras clave: Educación primaria, metodología activa, proyecto educativo, tiempo histórico, trabajo cooperativo.

\section{Recibido: 14/V/2020. Aceptado: X/2020}

1 Este trabajo nace en el marco del proyecto de mejora docente de la Universidad de Córdoba: "Construir la Historia a través de las competencias educativas en Educación Primaria" (2017-1-4012), y también se incluye en el Proyecto de Investigación PGC2018-097481-B-I00, financiado por el Ministerio de Ciencia, Innovación y Universidades de España. 


\begin{abstract}
This work shows a didactic experience whose main objective has focused on incorporating teamwork dynamics with students in the fourth year of Primary Education and on acquiring educational skills to approach the origin and evolution of the human being, to the ways of life prehistoric, and to the cultural and artistic manifestations created by those first humans, in the longest period of Humanity. Through the implementation of an investigative methodology, historical events related to the period spanning from the Paleolithic to the Metal Age were analyzed and it was sought to strengthen the historical capacities of the students with the performance of exercises that worked on historical time and the chronological space where the most relevant events of Prehistory took place. The results of its implementation showed a student body capable of analyzing and interpreting events with historical coherence and perspective. The conclusions drawn by the proposal stand out for cooperative work as an instrument to guarantee the learning of inquiry and knowledge of prehistoric heritage.
\end{abstract}

Keywords: Primary Education, active methodology, educational project, historical time, cooperative work.

\title{
1. INTRODUCCIÓN
}

En el desarrollo de esta propuesta titulada «La vida en la Prehistoria», la experiencia previa del alumnado y las situaciones reales que han vivido serán el principio didáctico de partida, así como la adecuación de los aprendizajes. Al respecto Chaves indica: «Para Vigotsky las escuelas representaban los mejores «laboratorios culturales» para estudiar el pensamiento y modificarlo mediante la acción cooperativa entre adultos e infantes» (2001: 61). Desde esta perspectiva, el enfoque histórico-cultural de esta experiencia potencia en el proceso de desarrollo del alumnado no solamente los contenidos culturales, sino también los procedimientos de pensamiento históricos.

En este sentido, son los intereses del alumnado de Educación Primaria los que refuerzan el aprendizaje de la didáctica de las ciencias sociales, al ser una materia que comparte líneas temáticas con el estudio del espacio geográfico y del tiempo histórico (Palma, 2013). Así, como afirma García-Valcárcel y Basilotta: «Es necesario que el proyecto esté centrado en el estudiante, adaptado a sus necesidades e intereses, despierte su curiosidad y genere motivación» (2017: 115). Es aquí donde la divulgación arqueológica es beneficiosa para el conjunto de la sociedad (Ruiz-Zapatero, 2009), además de presentar la ventaja de manipular y hacer una toma de contacto con los objetos prehistóricos en diferentes niveles educativos (Camarós, 2009).

Por su parte, la aportación de Kolmos (2004: 85), reafirma que el trabajo por proyectos se entiende como una manera de organizar el proceso de aprendizaje centrada en la formulación de una problemática. De ahí que la resolución de problemas favorezca el desarrollo personal para el autoaprendizaje (Lerís y Sein-Echaluce, 2011). Sin embargo, Pagès y Santisteban (2010: 285) consideran que al finalizar la escolaridad obligatoria los aprendizajes del alumnado sobre la historia están formados por una serie de elementos aislados, hechos, personajes y fechas. De hecho, en la etapa de Primaria se trabajan aspectos abstractos como la temporalidad prehistórica que es lo más alejado a lo concreto del espacio vivido y cotidiano del alumnado. 
Ahora bien, la enseñanza de la Historia siempre ha sido un elemento clave en la didáctica de las ciencias sociales y se torna imprescindible para dar respuestas a muchas preguntas que se plantean de la vida diaria (Suárez Suárez, 2013; García González y Gabriela, 2014). Pero su enseñanza no siempre se percibe por el alumnado como se debiese, ya que sus contenidos suponen un establecimiento de ideas que en muchos casos son difíciles de entender e interpretar. En cierto modo, los periodos en el tiempo, el concepto abstracto de temporalidad, así como otros errores asociados a su enseñanza como la causalidad de acontecimientos, son unos de los mayores retos que los docentes deben incorporar a su formación para que el alumnado pueda comprender y aprender la materia y la adquisición de diversas habilidades (Sáiz Serrano, 2013).

En esta dirección, la propuesta didáctica implementada fomenta la capacidad de análisis del pasado, además de plantear interrogantes investigativos que les acerquen a los procesos de aprendizaje de la Historia. Tal y como afirman López-Castilla, Terradillos-Bernal y Alonso (2017: 358), la Empatía Prehistórica supone el desarrollo de una metodología activa que involucra al público en general en el desarrollo de las características técnicas identificadas en la Prehistoria. Acertadamente la aportación de San Martín y Ortega-Sánchez (2020: 9), evidencia que, al trabajar la empatía prehistórica, incorporando la dimensión emocional, se contribuiría a humanizar el fenómeno prehistórico. En esta línea, gracias a la arqueología y su grado de experimentalidad motivamos la implicación y el aprendizaje activo del alumnado (MolinaTorres, Niveau-de-Villedary y López, 2020).

Por último, debemos destacar que este trabajo nace en el marco del proyecto de mejora docente de la Universidad de Córdoba: «Construir la Historia a través de las competencias educativas en Educación Primaria» (2017-1-4012), y también se incluye en el Proyecto de Investigación PGC2018-097481-B-I00, financiado por el Ministerio de Ciencia, Innovación y Universidades de España.

\section{OBJETIVOS Y COMPETENCIAS EDUCATIVAS}

El objetivo principal de esta experiencia se centra en facilitar la enseñanza-aprendizaje del periodo histórico escogido, la Prehistoria, y hacerlo desde un punto de vista globalizador, cooperativo, interdisciplinar e inclusivo. Para dar respuesta a este objetivo, se establecieron otros objetivos específicos para la consecución de la propuesta didáctica:

1. Conocer las formas de vida prehistóricas.

2. Descubrir la relación que existe entre el pasado y el presente.

3. Formular hipótesis sobre las diferencias y semejanzas de los grupos humanos de la Prehistoria y nuestros hábitos actuales.

4. Investigar los grupos sociales prehistóricos, además de sus creencias, viviendas, alimentación, etc.

5. Poner en práctica actitudes colaborativas y de trabajo grupal.

6. Utilizar diferentes recursos y herramientas digitales. 
A este respecto la temática trabajada, la Prehistoria, queda perfectamente contextualizada según el Real Decreto 126/2014, de 28 de febrero, por el que se establece el currículo básico de la Educación Primaria (BOE, 52, de 1 de marzo de 2014). No obstante, los contenidos curriculares introducidos en el citado Real Decreto se corresponden parcialmente con el periodo de nuestra propuesta, ya que éstos se ciñen exclusivamente a la Península Ibérica, sin que podamos hacer alusión a los acontecimientos históricos que desarrollamos desde una perspectiva de conjunto más amplia, cronológica y espacialmente. A continuación, se detallan los contenidos relativos a la etapa histórica, los estándares de aprendizaje y los criterios de evaluación dirigidos a $4^{\circ}$ de Educación Primaria.

Tabla 1. Contenidos, criterios de evaluación y estándares de aprendizaje en los que se encuadra la propuesta.

\begin{tabular}{|l|l|l|}
\hline \multicolumn{1}{|c|}{ Contenidos } & \multicolumn{1}{|c|}{ Criterios de evaluación } & \multicolumn{1}{c|}{$\begin{array}{c}\text { Estándares de aprendizaje } \\
\text { evaluables }\end{array}$} \\
\hline El tiempo histórico y su medida. & $\begin{array}{l}\text { 4. Desarrollar la curiosidad } \\
\text { por conocer las formas de vida } \\
\text { humana en el pasado, valorando la } \\
\text { importancia que tienen los restos } \\
\text { clasificación. }\end{array}$ & $\begin{array}{l}\text { 3.5. Explica la diferencia de los } \\
\text { dos períodos en los que se divide } \\
\text { la Prehistoria y describe las } \\
\text { características básicas de las formas de } \\
\text { La Península Ibérica en la Prehistorianiento y estudio de la } \\
\text { historia y como patrimonio cultural } \\
\text { que hay que cuidar y legar. }\end{array}$ \\
\hline
\end{tabular}

Fuente: elaboración propia.

Del mismo modo, para el desarrollo de la propuesta diseñada se trabajaron en el aula las siete competencias clave, aunque se prestó especial atención a las tres siguientes: la competencia en comunicación lingüística, la digital, además de la competencia relativa a la conciencia y expresiones culturales.

Tabla 2. Competencias educativas y características de los recursos que se implementaron en la propuesta.

\begin{tabular}{|l|l|}
\hline \multicolumn{1}{|c|}{ Competencias clave } & \multicolumn{1}{c|}{ Características } \\
\hline Conciencia y expresiones culturales. & $\begin{array}{l}\text { Íntimamente relacionada con los contenidos del tema que versan sobre } \\
\text { las manifestaciones más relevantes del arte prehistórico y las cuestiones } \\
\text { relacionadas con el mundo de las creencias de las sociedades prehistóricas. }\end{array}$ \\
\hline Digital. & $\begin{array}{l}\text { Se fomenta la búsqueda de información a través de Internet. Se hizo } \\
\text { un análisis crítico de la información obtenida a través de los buscadores, } \\
\text { servidores o portales de la red (Google, YouTube, etc.), además de utilizar } \\
\text { el blog como un recurso de aula necesario para transmitir conocimientos y } \\
\text { favorecer el interés por la historia. }\end{array}$ \\
\hline Comunicación lingüística. & $\begin{array}{l}\text { Se trabajó mediante la realización de pequeños debates, en los que la docente } \\
\text { propondrá a los/as alumnos/as que expresen de manera razonada sus propios } \\
\text { puntos de vista sobre los temas objeto de estudio. }\end{array}$ \\
\hline
\end{tabular}

Fuente: elaboración propia. 


\section{METODOLOGÍA}

\subsection{Participantes y muestra}

Partimos de la base de que toda propuesta didáctica debe estar justificada en función del curso programado y la materia que se planifica, en nuestro caso ciencias sociales, y sin duda el contexto sociocultural en el que se aplica, un centro docente público andaluz, en el que se imparten Educación Infantil y Primaria, contabilizándose en la muestra de estudio cuatro grupos de $4^{\circ}$ de Primaria $(n=108)$. Asimismo, en el centro se llevan a cabo una serie de programas que tendrán su reflejo en esta propuesta y en las actividades que en ella se plantean: Plan de Igualdad entre Hombres y Mujeres en Educación, Plan de Convivencia, Plan de Fomento de Lectura y Biblioteca. También al ser éste un centro TIC, el uso de las nuevas tecnologías será uno de nuestros objetivos (Carrió, 2007).

\subsection{Diseño}

En el estudio se utilizó una metodología cualitativa a través de la observación directa, continua y sistemática. Para su realización se necesitó de una inmersión prolongada, intensiva y directa del docente en las actividades del grupo (Latorre, Rincón y Arnal, 2005). En este sentido, atendiendo al enriquecimiento que se produce en el aprendizaje mediante la interacción de los/as estudiantes, se fomentaron dinámicas en las que se trabaje en pequeños grupos heterogéneos o por parejas, y también con actividades individuales o en gran grupo. Con estos agrupamientos se favoreció la autonomía, el trabajo colaborativo y la configuración de grupos de apoyo interactivos (Quinquer, 2004; Clark y Baker, 2009; Avello-Martínez y Marín, 2015), de manera que el alumnado asuma la planificación y ejecución del proyecto en diferentes fases, se analicen los contenidos de manera consensuada y adquieran una serie de estrategias pedagógicas para aprender de formar autónoma. Así, procuramos que se promueva el aprendizaje significativo y se desarrollen las clases, principalmente, a través de interacciones con el mundo exterior para facilitar la adquisición de las competencias educativas (Cano, 2005).

\subsection{Desarrollo de la experiencia}

En el proceso de enseñanza y aprendizaje de la historia, existen varios factores para tener en cuenta a la hora de identificar el origen de las dificultades de aprendizaje del alumnado. Y es que estos problemas de aprendizaje no solo se derivan de la naturaleza propia del campo de las ciencias sociales, sino que también tienen que ver con los diseños curriculares establecidos, los métodos de enseñanza impartidos o incluso de los recursos y materiales que se empleen en su didáctica. Para la elaboración de la propuesta hemos tenido en cuenta las experiencias personales del alumnado para trabajar el patrimonio arqueológico de nuestra ciudad y enfocar las fases de la planificación docente con una perspectiva globalizadora e interdisciplinar (Cuenca, 2014; Molina-Torres, 2018). En relación con estas aportaciones, Díaz Villafáñez (2016: 44) expone que:

Ningún aprendizaje en cualquier etapa educativa que pensemos, aunque todavía más en la de primaria, por la importancia de partir de unos conocimientos adquiridos y hacerles vivenciales, no podemos separarles por materias, sino que tenemos que englobarles en un proyecto integral 
globalizador para darles un sentido relevante y verdaderamente significativo para el escolar de primaria.

Dentro de esta metodología se utilizó un portafolio digital en el blog de aula para describir, sentir, comprender, explicar y reconstruir la historia. Esta herramienta estimuló la evaluación por pares y la autoevaluación, proporcionando información permanente y actualizada no solo del alumnado, sino también del docente. De hecho, los principios clave para su elaboración e implementación se centraron en fortalecer la autonomía, la reflexión la tutorización grupal y el marco de competencias educativas.

En relación con el panorama que hemos descrito, Caballero (2000: 78-79) indica que dentro del planteamiento metodológico caben reseñar algunos elementos afines con la enseñanza de las ciencias sociales, entre los que destacan:

1. Sondear la situación de partida del alumnado: con una prueba inicial que permita obtener un diagnóstico certero del contexto, para conocer si dominan técnicas básicas para el desarrollo de la materia que se va a impartir, si manejan conceptos esenciales en la misma o si están habituados a ciertas técnicas de trabajo.

2. Explorar las ideas previas de los/as estudiantes: en cada núcleo de contenidos, dedicando a ello una sesión lectiva o, al menos, una parte de ésta. Emplearemos para la exploración la técnica del torbellino de ideas, mediante la cual la docente lanza una pregunta motivacional y el alumnado aporta sus puntos de vista y reflexiones que fueron anotando resumidamente en la pizarra.

3. Establecer un clima de aula satisfactorio: se trata de que los niños y niñas nos perciban con alguna proximidad, considerando al profesor/a como alguien que pueda orientarlos en su trabajo y aclararles las dudas que vayan surgiendo. Esta situación propició su interés hacia la asignatura y, en consecuencia, estimuló los aprendizajes.

4. Combinar el trabajo individual y el grupal: se combinarán secuencias de trabajo individual con otras de trabajo en grupo. Para ello se tendrán en cuenta la colaboración, el reparto de tareas entre los miembros del pequeño grupo, así como las capacidades personales.

5. Introducir cambios de ritmo: desarrollo de diversos ritmos de trabajo durante las fases de realización del proyecto (motivación inicial, implementación de la propuesta y resumen de lo aprendido).

6. Proporcionar el uso de fuentes diversas: procuraremos evitar que el libro de texto que utilicemos se convierta en la única herramienta didáctica. Así pues, plantearemos el empleo de fuentes históricas y geográficas combinando materiales elaborados por los docentes o que se encuentran en la biblioteca del aula y recursos webs, aunque siempre bajo la supervisión del docente, que ha de enseñar también al alumnado a incluir, en sus trabajos, las referencias a las fuentes empleadas.

7. Fomentar las salidas del aula: hacer salidas de campo para ponerlos en contacto directo con el entorno y aprovechar los contenidos que se están trabajando en el aula. 
8. Disponer el aula como un espacio inclusivo: tratar de atender, en la medida de lo posible, la diversidad de aprendizajes en la escuela. Uno de los aspectos más importantes que debemos incluir como estrategia inclusiva serían los grupos heterogéneos de apoyo.

9. Propiciar la obtención de conclusiones personales y favorecer el pensamiento autónomo: para ello se emplean la realización de síntesis, de tramas conceptuales, los debates en el grupo-clase, etc.

10. Dotar a la enseñanza de un sentido activo: se trata de conseguir que el alumno se vea a sí mismo/a como un agente implicado en el desarrollo de sus facultades intelectuales, mostrando interés por poner en juego su propia capacidad para progresar. Y para ello, en definitiva, los docentes debemos abandonar los modelos unidireccionales que relegan al alumno y alumna al papel de simple oyente de nuestro discurso científico.

En este sentido, es imprescindible tener en cuenta la diversidad del alumnado a la hora de planificar las actividades y organizar el trabajo. De hecho, no se nos escapa la dificultad que supone, en aulas relativamente numerosas, trabajar simultáneamente con diferentes ritmos o con actividades de diferente tipo. Por ello, y dependiendo de las necesidades del alumno/a, podrán establecerse actividades de ampliación o de refuerzo para aquellos que así lo precisen. Las actividades de refuerzo sirvieron para repasar los contenidos tratados en la propuesta, revisando sus aspectos más importantes como serán esquemas, resúmenes, mapas conceptuales; mientras que las actividades de ampliación permitieron al alumnado profundizar en estos contenidos realizando, por ejemplo, pequeñas investigaciones a través de Internet sobre aspectos relevantes de la misma. Todas estas actividades estuvieron personalizadas y se realizaron en horario no lectivo, utilizando para ello el blog de aula.

Evidentemente, las actividades que se planificaron al comienzo del proyecto fueron organizadas por el alumnado mediante el trabajo colaborativo en el aula y fuera de ésta. De manera que la organización en pequeños grupos de trabajo de cuatro o cinco alumnos y alumnas fuese paritaria y con ello se promueva la motivación e implicación en el proyecto. En este contexto, la docente hizo de guía y orientó la actividad al inicio para ir delegando responsabilidades gradualmente. Las tareas, enfocadas a las presentaciones de los equipos, se establecieron siguiendo unos roles preestablecidos en varias fases:

\section{Fase de inicio a la investigación (1 sesión)}

En primer lugar, hicimos un sondeo al alumnado sobre la Prehistoria, con preguntas como: ¿Qué cosas conocéis sobre la época prehistórica? A modo de resumen, se anotaron en la pizarra las observaciones formuladas por los/as estudiantes. Posteriormente, la docente comenzó un breve diálogo sobre estas cuestiones: ¿Cómo vivían los hombres y las mujeres en la Prehistoria? ¿Eran nómadas o sedentarios/as? ¿Por qué? ¿Cuánto duró la Prehistoria? ¿Y todo el tiempo de la Prehistoria los hombres y las mujeres vivieron de la misma manera? 
Esta primera sesión también incluyó la presentación de una actividad investigativa a través de la cual el alumnado tuvo la oportunidad de ir conociendo un poco más acerca del entorno de su ciudad. Para esta actividad, en concreto, imaginaron a un grupo social del Paleolítico que vivió en el entorno del Guadalquivir, y se plantearon las siguientes cuestiones: ¿De qué se alimentaban y de qué modo conseguían los alimentos? ¿Crees que ha cambiado el entorno ambiental de esas personas con el actual?

\section{Fase de implementación (5 sesiones)}

Se originó un feedback entre alumnado y profesor para establecer la resolución de problemas que fueron surgiendo durante el aprendizaje experiencial. Para ello, facilitamos la interacción y el aprendizaje colaborativo para hacer este intercambio de ideas grupales. Del mismo modo, para fomentar la creatividad utilizaron un blog de aula para colgar la experiencia educativa (figura 1).

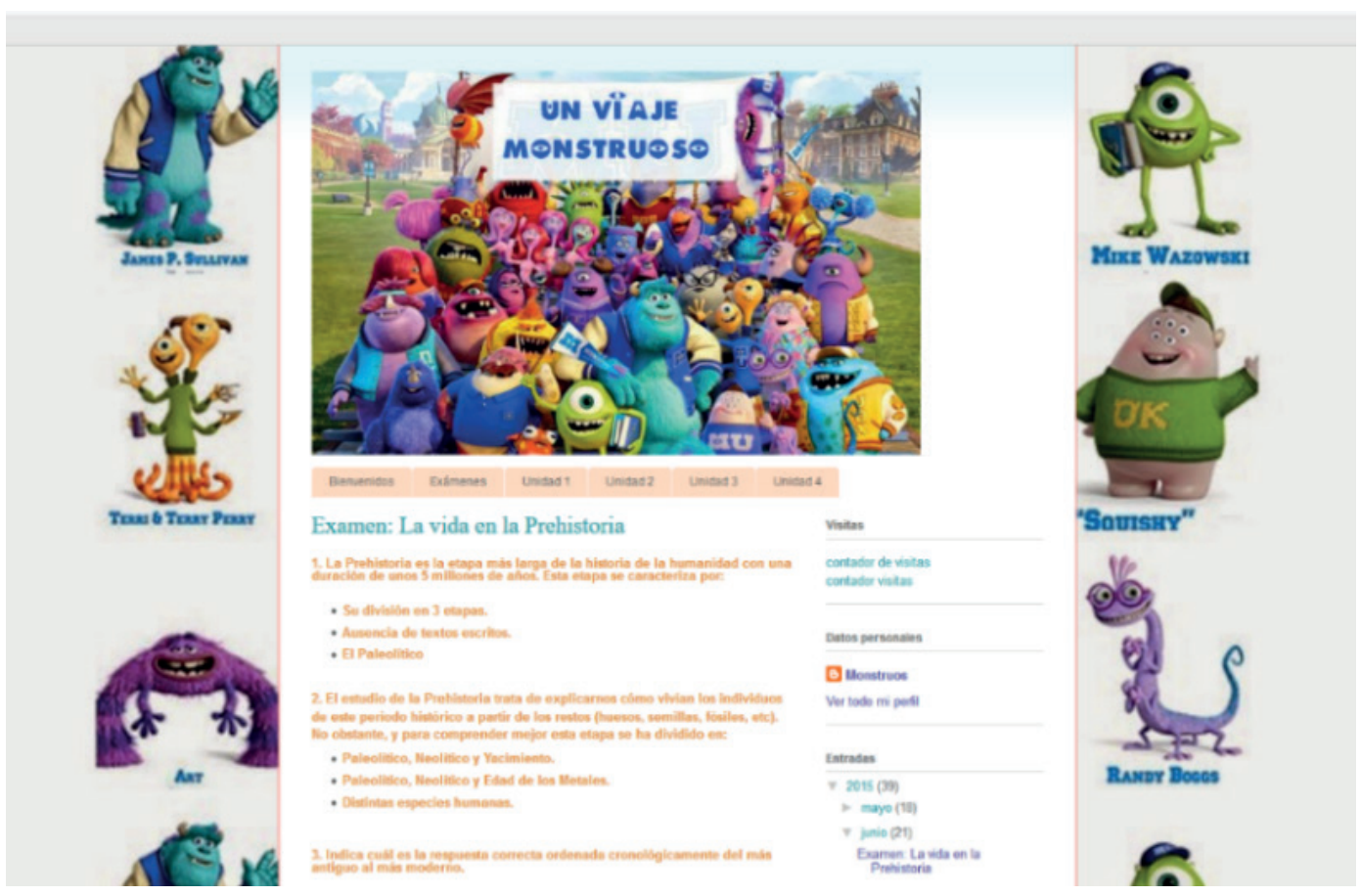

Figura 1. Blog de aula utilizado para la propuesta didáctica.

Fuente: http://unviajemonstruoso.blogspot.com/2015/05/examen-prehistoria.html

Para el bloque de contenidos sobre «El origen de la Humanidad», la docente pidió a un/a alumno/a que lea, de su libro de texto y en voz alta, un documento que presenta, en pocas palabras, una panorámica-resumen. A continuación, se planteó una primera actividad centrada en la definición del concepto de «proceso de hominización», que los niños y las niñas 
anotaron en su libreta de vocabulario. Una segunda actividad se centró en completar el eje cronológico del proceso de hominización, en el que aparecen los siguientes términos: Australopitecus, Homo Habilis, Homo Erectus, Homo Antecessor, Neandertal, Homo Sapiens. Con la representación de este friso lineal pudimos reflejar el lenguaje estereotipado de los libros de texto y la construcción de la (pre)historia con una perspectiva androcéntrica.

Del mismo modo, para conocer más sobre la vida en el Paleolítico, en primer lugar, se realizó una introducción general que la docente efectuó mediante una exposición oral de aproximadamente diez minutos de duración. A continuación, se redactó un ejercicio narrativo acerca de los avances tecnológicos que tuvieron lugar a lo largo del Paleolítico, centrando la atención en varias cuestiones básicas, como el instrumental, el uso del fuego, las actividades depredadoras y la organización social en el Paleolítico. En este caso concreto, se trató de que los/as estudiantes pudieran a través de sus propios testimonios profundizar en contenidos de forma más autónoma. La principal motivación fue una investigación sobre quién fue Lucy: ¿a qué especie perteneció?, ¿cuándo fue descubierta?, ¿dónde?, ¿por qué le pusieron ese nombre? Para ello, se les proporcionó la siguiente página web:

\section{http://www.portalciencia.net/antroevoafar.html}

Asimismo, para documentar «La revolución Neolítica», se solicitó a un/a alumno/a que leyera en voz alta, de su libro de clase, un texto sobre las actividades económicas que se iniciaron en el Neolítico, agricultura y ganadería. A continuación, se les preguntó sobre las posibles ventajas y desventajas de la realización de estas nuevas actividades productoras que suscitó un breve debate para fijar los aspectos de mayor relevancia de la economía neolítica y su organización socioeconómica. Seguidamente, se aprovechó la sesión para tratar algunos aspectos relativos a la vida en la Edad de los Metales. Además, se proporcionó al alumnado algunas direcciones de Internet en las que pudieron localizar informaciones específicas sobre varios de los temas tratados en las clases anteriores. A modo de ejemplo se presenta el siguiente enlace web:

http://www.ite.educacion.es/w3/eos/MaterialesEducativos/mem2001/huellas/origenes/main.html

Por último, se corrigió en clase el mapa conceptual que copiaron en la primera sesión. En pequeños grupos fueron definiendo los distintos conceptos que aparecen en éste. Se recomendó a los/as estudiantes que colorearan cada aspecto con un color diferente y que explicaran, apoyándose en las flechas de dirección existentes en el esquema, las relaciones de dependencia entre unos conceptos y otros, distinguiendo en cada fenómeno sus causas y sus consecuencias. Los conceptos del esquema estaban presentados de forma que permitían retomar las cuestiones analizadas a lo largo de la propuesta, de tal modo que dicho mapa conceptual sirviese, no sólo para sintetizar las ideas básicas, sino también para afianzar los conocimientos adquiridos. A continuación, y tras la elaboración de las distintas actividades, registraron de manera individual aquello que habían aprendido en su diario de aprendizaje, las tareas que más les habían gustado y aquellas que les resultaron más difíciles de realizar. 


\section{Fase de síntesis y recapitulación de ideas (2 sesiones)}

Llegados a este punto nuestra investigación dio paso a una práctica de campo para conocer los modos de vida en la Prehistoria. Para documentar al alumnado hicimos una visita guiada al Museo Arqueológico de Córdoba con el propósito de observar y analizar la cultura material que aparece expuesta en las vitrinas del edificio. A través de la arqueología experimental pudimos hacer herramientas líticas y diversos utensilios de caza y pesca, con el objetivo de conocer las técnicas para su fabricación y los materiales utilizados, saber en que creían mediante el conocimiento de los ídolos calcolíticos, dónde vivían en las distintas etapas de la Prehistoria, cómo organizaban los poblados.

También se propuso, a modo de resumen, la realización de una actividad de indagación: Conoce tu entorno. A través de esta actividad que realizaron en pequeños grupos (4 o 5 alumnos y alumnas), descubrieron más de cerca el entorno de su ciudad y tomándola como base aplicaron sus conocimientos de Cartografía (elaborando un mapa de Córdoba y localizando la ciudad a través de las coordenadas), Geografía Física (diseñando el relieve y los ríos de su entorno, situándolos en el mapa, deduciendo por su situación sus características climáticas generales, describiendo sus formaciones vegetales, etc.), e Historia (elaboración de redacciones sobre un día en la vida de una familia del Paleolítico, además de ir descubriendo las culturas antiguas que han pasado por la localidad y las relaciones que mantuvieron sus habitantes con las civilizaciones del Mediterráneo).

Esta actividad permitió recopilar todo lo aprendido, revisando y reforzando los contenidos, y permitiendo la expresión de la creatividad y de la imaginación del grupo-clase. Es una forma de incorporar los aprendizajes obtenidos al entorno más cercano del alumnado. Cada grupo realizó un mural al que incorporaron textos, fotos, dibujos, etc. Estos murales se colgaron en la pared del aula. Asimismo, en el desarrollo de los contenidos programados se prestó especial atención al estudio de la situación de la mujer, contribuyendo así al Plan de Igualdad entre Hombres y Mujeres en Educación. Concretamente se elaboró una actividad para investigar y elaborar, en pequeños grupos, un mural que incluya textos y fotos que fueron acompañadas de narrativas sobre los roles y los estereotipos de género que existían en la Prehistoria (Pastor y Mateo, 2019), y la invisibilidad de las mujeres en este periodo de la historia.

\subsection{Instrumento para la recogida de datos}

Para la recogida de información se plantearon tres cuestiones abiertas de fácil comprensión que sirvieron para orientar los resultados del estudio y dar respuesta a los objetivos fijados en el mismo (Flick, 2007; Gibbs, 2012). Aunque el alumnado respondió a cada una de ellas de manera individualizada, las actividades prácticas, la puesta en común durante las sesiones de trabajo y el aprendizaje colaborativo se desarrollaron con carácter grupal. Para ello, respondieron a las siguientes preguntas:

1. ¿La forma de vida en la Prehistoria me parece distinta a la nuestra?

2. ¿Sé diferenciar las tareas que realizaban mujeres y hombres en la Prehistoria?

3. ¿El blog de aula me ha servido para conocer y compartir los trabajos de mis compañeros y compañeras de clase? 


\section{RESULTADOS}

Los resultados de la evaluación cualitativa reflejan las percepciones del alumnado de Educación Primaria sobre los modos de vida en la Prehistoria y su comparativa con las costumbres actuales. Las respuestas de esta primera cuestión hacen referencia a diferentes versiones de esta situación, destacando: «pienso que nosotros(as) no vivimos como en la Prehistoria» (alu_028, alu_068); «los vestidos y la comida no son lo mismo que tenemos ahora» (alu_21); «me gusta vivir mejor en esta época, porque en la Prehistoria todo sería más complicado» (alu_34; alu_88); «¿los(as) niños(as) prehistóricos(as) iban a la escuela?» (alu_64; alu_13).

En lo que respecta a la segunda cuestión, el alumnado reproduce cierta confusión para diferenciar los roles femeninos de los masculinos. Nos dicen: «ilas mujeres solo cuidan de los(as) hijos(as) y no cazaban?» (alu_81; alu_101); «los hombres y las mujeres hacen cosas diferentes... los primeros cazan y siempre van juntos, y ellas solo están cuidando de los bebes» (alu_43; alu_56); «la mujer cuida de su familia y el hombre trae comida, no hacen lo mismo» (alu_30; alu_12).

En la última cuestión indicaban que: «el blog me ha servido para estudiar con mi grupo de compañeros/as» (alu_105); «me ha gustado mucho trabajar así con un blog» (alu_84); 《en otras asignaturas no lo utilizamos (el blog)...lo prefiero usar para aprender» (alu_17); «ha sido muy divertido compartir las actividades de mi grupo con otros grupos de mi clase» (alu_55; alu_44); «además he podido ver todas las tareas que hemos realizado entre todos/as» (alu_73).

Analizadas las respuestas se muestra una comparativa de la Prehistoria y la actualidad donde el alumnado aprecia marcadas diferencias entre las dos épocas históricas. En cuanto a los roles femeninos y masculinos, tienen claras las diferenciaciones sociales y económicas que son asimiladas por ambos géneros. Por último, la utilización del blog en el aula les ha parecido útil e interesante por varios motivos como visualizar las actividades, compartirlas con el resto de los grupos y poder organizar las tareas para colgarlas conjuntamente.

\section{CONCLUSIONES}

Tras la ejecución del proyecto se comprobó que la investigación de la temática elegida promovió el aprendizaje por descubrimiento, el conocimiento y valoración del patrimonio arqueológico y prehistórico, y la concienciación de la conservación de éste. Es así como durante todas las fases se establece una planificación flexible y abierta que determine la multiplicidad de aprendizajes. Con ello, se constató una mejora generalizada del rendimiento y de la motivación de los alumnos y las alumnas, además de la puesta en práctica del aprendizaje cooperativo y la construcción colectiva del conocimiento (Slavin, 2014; Gillies, 2016).

Respecto al considerable aprendizaje derivado de la experiencia, es reseñable la evidente mejoría del alumnado para dejar de lado la memorización y adquirir unas habilidades cognitivas que potencien el conocimiento conceptual, la reflexión propia y el análisis del entorno que nos rodea. Al respecto aportaciones como las de Sanahuja, Peiro y Piquer (2015), indican 
que la metodología por proyectos para trabajar la Prehistoria ofrece diferentes perspectivas de aprendizaje, garantizando las necesidades y también diferentes estilos de enseñanza en el alumnado.

Partiendo de estas premisas, debemos incidir en la evaluación a través de tres preguntas de respuesta abierta con el propósito de mejorar las estrategias utilizadas y la obtención de los resultados de nuestra experiencia. En esta línea, Garrido (2010) subraya la importancia de participar en la manipulación de la cultura material que nos conecta con los modos de vida de otras épocas históricas, los restos cerámicos, las herramientas para la recolección que se fabricaban en aquellos tiempos. El estudio de Affuso y Preite (2009) muestra una experiencia en un colegio de Primaria en Italia donde la arqueología experimental supone una oportunidad educativa para el conocimiento de las estrategias didácticas que faciliten el proceso práctico de enseñanza y aprendizaje. De este modo, el presente proyecto de innovación educativa abre nuevas líneas de acción y mejora para integrar el aprendizaje por descubrimiento en una realidad de aula práctica y renovada.

Por lo que respecta al producto final se desenvolvió al mismo tiempo que la investigación y el análisis de la propuesta. De hecho, las actividades realizadas durante todo el proceso fueron pensadas cuidadosamente de modo que con cada una se alcanzara un objetivo específico. Sin embargo, durante todo el recorrido didáctico hemos detectado fortalezas y debilidades que muestran los beneficios y las limitaciones del proyecto (tabla 3). Entre las primeras, destacan las situaciones de aprendizaje entre iguales que propician el aprendizaje colaborativo y cooperativo, así como las tareas competenciales que quedaron definidas tanto por el alumnado como por el profesorado, lo que mejoró la interacción entre los compañeros y el fomento de la inclusión (Majó, 2010; Traver y Rodríguez Feliu, 2011; Johnson y Johnson, 2014; Lata y Castro, 2016).

En cuanto a las debilidades se centraron en la desconfianza del alumnado hacia un método de enseñanza del que desconocen su implementación, al estar acostumbrados a una metodología transmisiva, lo que puede provocar la falta de motivación de los/as estudiantes al emprender un proyecto del que ignoran la temática y las fases de un trabajo esencialmente investigativo al que no están habituados en otras asignaturas. Para ello Santiago Asensio (2013) piensa que el trabajo por proyectos se encuentra no solo en la motivación de los docentes, sino también en la de los alumnos. Fue este cambio de metodología lo que provocó que el alumnado asuma la ejecución y planificación de todo el proceso, que adquieran competencias y estrategias que les ayuden a aprender, organizar, analizar una etapa histórica, elaborar recursos y confiar en sus capacidades y posibilidades, así como acercar los avances arqueológicos e históricos a los centros escolares (Boj, 2005). 
Tabla 3. Análisis DAFO con las debilidades y fortalezas de la propuesta didáctica.

\begin{tabular}{|l|l|}
\hline \multicolumn{1}{|c|}{ Debilidades } & \multicolumn{1}{c|}{ Amenazas } \\
\hline $\begin{array}{l}\text { Ausencia, en ocasiones, de formación de los docentes y } \\
\text { también de motivación para utilizar estrategias educativas } \\
\text { que fomenten el trabajo colaborativo. }\end{array}$ & $\begin{array}{l}\text { Las familias desconfían de este método al no tener un } \\
\text { libro de texto que dirija el aprendizaje de sus hijos/as. } \\
\text { Falta de coordinación con los equipos docentes. }\end{array}$ \\
\hline \multicolumn{1}{|c|}{ Fortalezas } & \multicolumn{1}{c|}{ Oportunidades } \\
\hline $\begin{array}{l}\text { Implicación y participación de los/as estudiantes con } \\
\text { una metodología que no han puesto en práctica en otras } \\
\text { materias. Mejora de la interacción entre compañeros y } \\
\text { fomento de la inclusión. }\end{array}$ & $\begin{array}{l}\text { Esta experiencia supone una evaluación centrada } \\
\text { más en las competencias que en los contenidos. Y } \\
\text { también una adecuada interrelación entre áreas de } \\
\text { conocimiento. }\end{array}$ \\
\hline
\end{tabular}

Fuente: elaboración propia.

En definitiva, esta experiencia educativa nos ha servido para implementar una propuesta de mejora a las nuevas metodologías de enseñanza y desarrollar una iniciativa didáctica que presente a la Prehistoria como una disciplina interesante y llamativa para el alumnado de Educación Primaria. Sin embargo, que esta disciplina ocupe un lugar secundario en la normativa educativa perjudica su puesta en valor tanto histórica como patrimonialmente. Esta situación muestra una implicación necesaria de la intervención docente en la educación prehistórica, debido a que es un periodo en el que no se incide tanto en el aula, donde tiene un tratamiento reducido en el currículo.

\section{REFERENCIAS BIBLIOGRÁFICAS}

Affuso, A. y Preite, A. (2009). Prehistory in school didactics. EuroREA, (6), 13-16.

Avello-Martínez, R. y Marín, V. (2015). La necesaria formación de los docentes en aprendizaje colaborativo. Profesorado. Revista de curriculum y formación del profesorado, 20(3), 687713.

Boj, I. (2005). El tratamiento didáctico de la prehistoria y la evolución humana. El caso de Atapuerca. Aula historia abierta, (15), 37-53.

Caballero, J. D. (2000). Didáctica de las Ciencias Sociales, Geografía e Historia. Sevilla: Mad.

Camarós, E. (2009). Breves reflexiones en torno a los «juguetes prehistóricos» como herramientas pedagógicas. Estrat Crític, (3), 89-96.

Cano, E. (2005). Cómo mejorar las competencias de los docentes. Barcelona: Graó.

Carrió, M. L. (2007). Ventajas del uso de la tecnología en el aprendizaje colaborativo. Revista Iberoamericana de Educación, 41(4), 1-10.

Chaves, A. L. (2001). Implicaciones educativas de la teoría sociocultural de Vigotsky. Revista Educación 25(2), 59-65. DOI: https://doi.org/10.15517/revedu.v25i2.3581

Clark, J. y Baker, T. (2009). Research that works: a practical approach to student collaborative work. Wellington: Business Studies.

Cuenca, J. M. (2014). El papel del patrimonio en los centros educativos: hacia la socialización patrimonial. Tejuelo. Didáctica de la Lengua y la Literatura, (19), 76-96. Recuperado de http://rabida.uhu.es/dspace/handle/10272/7927 
Díaz Villafáñez, M. (2016). El aprendizaje del tiempo y su enseñanza en la educación primaria. Tabanque Revista pedagógica, (29), 43-68.

Flick, U. (2007). Introducción a la investigación cualitativa. Madrid: Ediciones Morata.

García González, S. M. y Gabriela, M. (2014). Categorización de preguntas formuladas antes y después de la enseñanza por indagación. Praxis \& Saber, 5(10), 75-91. DOI: https://doi. org/10.19053/22160159.3023

García-Valcárcel, A. y Basilotta, V. (2017). Aprendizaje Basado en Proyectos (ABP): evaluación desde la perspectiva de alumnos de Educación Primaria. Revista de Investigación Educativa, 35(1), 113-131. DOI: https://doi.org/10.6018/rie.35.1.246811

Garrido, C. (2010). El museo local como herramienta de difusión y didáctica del patrimonio arqueológico. Algunas reflexiones en torno al caso español. Posgrado y Sociedad, 10(2), 42-54. DOI: https://doi.org/10.22458/rpys.v10i2.1879

Gibbs, G. (2012). Análisis de datos cualitativos. Madrid: Ediciones Morata.

Gillies, R. M. (2016). Cooperative Learning: Review of Research and Practice. Australian Journal of Teacher Education, 41(3), 39-54. DOI: https://doi.org/10.14221/ajte.2016v41n3.3

Johnson, D. W. y Johnson, R. T. (2014). La evaluación en el aprendizaje cooperativo: cómo mejorar la evaluación individual a través del grupo. Madrid: SM.

Kolmos, A. (2004). Estrategias para desarrollar currículos basados en la formulación de problemas y organizados en base a proyectos. Educar, (33), 77-96. DOI: https://doi. org/10.5565/rev/educar.262

Lata, S. y Castro, M. (2016). El Aprendizaje Cooperativo, un camino hacia la inclusión educativa. Revista Complutense de Educación, 27(3), 1085-1101. DOI: https://doi.org/10.5209/ rev_RCED.2016.v27.n3.47441

Latorre, A., Rincón, D. y Arnal, J. (2005). Bases metodológicas de la investigación educativa. Barcelona: Ediciones Experiencia.

Lerís, D. y Sein-Echaluce, M. L. (2011). La personalización del aprendizaje: Un objetivo del paradigma educativo centrado en el aprendizaje. Arbor, 187(Extra_3), 123-134. DOI: https://doi.org/10.3989/arbor.2011.Extra-3n3135

López-Castilla, M. P., Terradillos-Bernal, M. y Alonso, R. A. (2017). The role of experimental archaeology in the scientific spreading as developer of prehistorical empathy. En R. Alonso, J. Baena y D. Canales (Eds.), Playing with the time: experimental archaeology and study of the past (pp. 355-360). Madrid: Servicio de Publicaciones de la Universidad Autónoma de Madrid.

Majó, F. (2010). Por los proyectos interdisciplinarios competenciales. Aula de Innovación Educativa, (195), 7-11.

Molina-Torres, M. P. (2018). La educación patrimonial en la Didáctica de las Ciencias Sociales en Primaria. Contextos Educativos. Revista de Educación, (22), 199-213. DOI: https:// doi.org/10.18172/con.3087

Molina-Torres, M. P., Niveau-de-Villedary, A. y López, N. (2020). El patrimonio histórico como recurso didáctico. La Ruta Fenicia de Gadir (Cádiz). Pulso. Revista de Educación, (43), 161-179. 
Pagès, J. y Santisteban, A. (2010). La enseñanza y el aprendizaje del tiempo histórico en la educación primaria. Cadernos Cedes, 30(82), 281-309. DOI: https://doi.org/10.1590/S010132622010000300002

Palma, A. (2013). Una propuesta didáctica para la enseñanza-aprendizaje del tiempo histórico y el espacio geográfico en el Grado de Maestro en Educación Primaria. Clío: History and History Teaching, (39), 1-16.

Pastor, M. y Mateo, D. (2019). Trabajo y roles de género durante la Prehistoria. Un estudio sobre su percepción en el alumnado de Historia. Panta Rei. Revista Digital de Ciencia y Didáctica de la Historia, 37-53. DOI: https://doi.org/10.6018/pantarei/2019/2

Quinquer, D. (2004). Estrategias metodológicas para enseñar y aprender ciencias sociales: interacción, cooperación y participación. Íber. Didáctica de las ciencias sociales, geografía e historia, (40), 7-22.

Ruiz-Zapatero, G. (2009). La divulgación arqueológica: las ideologías ocultas. Cuadernos de prehistoria y arqueología de la Universidad de Granada, (19), 11-36.

Sáiz Serrano, J. (2013). Alfabetización histórica y competencias básicas en libros de texto de historia y en aprendizaje de los estudiantes. Didáctica de las Ciencias Experimentales y Sociales, (27), 43-66.

San Martín, J. A. y Ortega-Sánchez, D. (2020). Empatía, empatía histórica y empatía prehistórica: una aproximación conceptual desde la enseñanza de las Ciencias Sociales. Didáctica de las Ciencias Experimentales y Sociales, (38), 3-16. DOI: https://doi.org/10.7203/ dces.38.15648

Sanahuja, A., Peiro, M. y Piquer, A. (2015). La prehistoria: Una experiencia de trabajo por proyectos en educación primaria. Quaderns digitals: Revista de Nuevas Tecnologías y Sociedad, (81), 6-23.

Santiago Asensio, P. (2013). El trabajo por proyectos: una forma de innovar en educación [Trabajo de Fin de Grado]. Valladolid: Universidad de Valladolid.

Slavin, R. (2014). Cooperative learning and academic achievement: Why does groupwork work? Anales de Psicología, 30(3), 785-791. DOI: https://doi.org/10.6018/analesps.30.3.201201

Suárez Suárez, M. A. (2013). Concepciones sobre la Historia en Primaria: La epistemología como asunto clave en la formación inicial de maestros. Didáctica de las Ciencias Experimentales y Sociales, (26), 73-93.

Traver, J. y Rodríguez Feliu, M. (2011). Los cuadernos de aprendizaje en grupo: una herramienta para favorecer el aprendizaje cooperativo. Valencia: Novadors. 